\title{
Spin Switch and Spin Amplifier: Magnetic Bipolar Transistor in the Saturation Regime
}

\author{
J. FABIAN \\ Institute for Theoretical Physics, Karl-Franzens University \\ Universitätsplatz 5, 8010 Graz, Austria \\ AND I. ŽUTIĆ \\ Center for Computational Materials Science, Naval Research Laboratory \\ Washington, D.C. 20375, USA \\ and \\ Condensed Matter Theory Center \\ Department of Physics, University of Maryland at College Park \\ College Park, Maryland 20742-4111, USA

\begin{abstract}
It is shown that magnetic bipolar transistors can amplify currents even in the saturation regime, in which both the emitter-base and collector-base junctions are forward biased. The collector current and the current gain can change sign as they depend on the relative orientation of the equilibrium spin in the base and on the nonequilibrium spin in the emitter and collector. The predicted phenomena should be useful for electrical detection of nonequilibrium spins in semiconductors, as well as for magnetic control of current amplification and for current switching.
\end{abstract}

PACS numbers: 72.25.Dc,72.25.Mk

\section{Introduction}

Silsbee's prediction [1] that nonequilibrium spin at an interface between normal and ferromagnetic metals gives rise to electromotive force (emf) was verified in the original Johnson-Silsbee spin injection experiment [2] with aluminum and 
permalloy. The observed voltages across the interface were on the order of picovolts, registering about one per $10^{11}$ spins in aluminum. An interesting application of the coupling was suggested by Johnson under the name of bipolar spin transistor [3] (polarity there refers to spin, not charge), or spin switch [4], an all metallic structure. In that proposal the current in the collector changes sign depending on the relative orientation of the two ferromagnets forming the switch. The scheme is explained in Fig. 1. Being all metallic, the device offers no current gain.

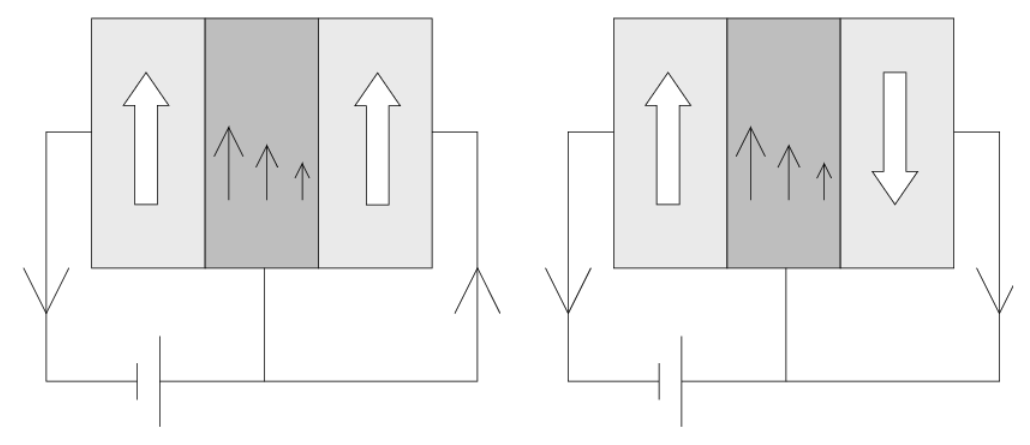

Fig. 1. Johnson's spin switch. In the metallic trilayer structure the two outer layers are ferromagnetic. Spin-polarized electrons are emitted from the left layer to the middle nonmagnetic metal. Assuming that spin polarization survives the diffusion to the right layer, the electrons either enter the right ferromagnet, if the magnetizations are parallel, or bounce back if the magnetizations are antiparallel. The spin-dependent emf appearing at the interface between the middle and right layers is due to the imbalance of the spinresolved chemical potentials. The current in the right ferromagnet (collector) can be switched by changing the relative magnetization orientation of the two ferromagnets.

We have shown that spin-charge coupling is significantly more pronounced in inhomogeneous magnetic semiconductors at nondegenerate doping levels $[5,6]$. By magnetic we mean either ferromagnetic (though these may be hard to make nondegenerate) or large-Zeeman effect (paramagnetic) semiconductors. The spin-charge coupling increases exponentially with applied bias, leading to, for example, giant magnetoresistance [5] (more than about 10\% change in the relative resistance in a magnetic multilayer structure with alternating magnetic and nonmagnetic layers, upon changing from parallel to antiparallel the magnetization of the magnetic layers).

Magnetic semiconductors integrated within the conventional device schemes show great technological potential. Several device schemes have been suggested that use magnetic semiconductors both as passive or active medium [7]. We have proposed to integrate magnetic transistors in the trilayer structure of a bipolar transistor, creating what we termed magnetic bipolar transistor [8] (MBT), in which any of the three regions (emitter, base, or collector) can be magnetic. 
In addition, nonequilibrium spin is assumed inside the structure, giving rise to spin-charge coupling. We have shown how in the active forward (the usual mode of operation) regime the current gain depends on the spin-charge coupling, leading to what we called giant magnetoamplification effect, which is a sensitive modulation of the charge current amplification by magnetic fields and nonequilibrium spin.

In this paper we describe other peculiar phenomena of MBT's: spin induced current gain and current switching (MBT as a Johnson spin valve transistor) in the saturation regime, which is usually the ON mode of the transistor in logic circuits. Conventional transistors, in contrast, show little current gain in this regime.

\section{Magnetic bipolar transistor}

The structure of a MBT is shown in Fig. 2. The transistor has three regions: emitter, base, and collector. In the scheme used here the emitter and collector are nonmagnetic, doped with donors ( $n$-doped), while the base is magnetic, doped with acceptors ( $p$-doped). By magnetic we mean that the carrier bands are spin split. For simplicity we consider only the conduction band to be spin split, leading to an equilibrium spin polarization of electrons. Holes are assumed unpolarized. The spin splitting can develop if the semiconductor is ferromagnetic, in which case the splitting is due to exchange coupling, or the semiconductor is doped with magnetic impurities and placed in a magnetic field, in which case the splitting arises from the large Zeeman effect [7]. For our purposes the origin of the splitting is not crucial, we describe what happens if the splitting occurs. One of the implications of such splitting is a finite spin polarization of the electron density $\alpha=\left(n_{\uparrow}-n_{\downarrow}\right) /\left(n_{\uparrow}+n_{\downarrow}\right)$, expressed in terms of the spin-resolved electron densities, $n_{\uparrow}, n_{\downarrow}$. We denote the equilibrium spin polarization in the base as $\alpha_{0 \mathrm{~b}}$, where the subscript " 0 " represents an equilibrium quantity. An important aspect of MBT is the possibility of having nonequilibrium spin in otherwise nonmagnetic regions. Spin can be introduced into the emitter or collector either optically [9] or electrically [10-13]. We denote the nonequilibrium (or excess) spin polarizations in the two regions by $\delta \alpha_{\mathrm{e}}$ and $\delta \alpha_{\mathrm{c}}$, respectively. An analytic theory of magnetic bipolar transistors was developed in Ref. [14] by generalizing Shockley's model [15].

We have previously described the physics of MBT's in the active forward regime $[8,14,16]$, in which the conventional bipolar transistor exhibits current gain. We have shown that the transistor exhibits magnetoamplification and giant magnetoamplification. The former arises from the trivial change of the equilibrium carrier density with the change in the carrier band splitting (see also Ref. [17]), while the latter appears due to spin-charge coupling. In the next section we describe the workings of MBT's in what is called the saturation regime, in which current gain in conventional transistors is limited, but in MBT's it can be large, with magnitudes as in the active forward regime. This effect is solely due to spincharge coupling. In order to understand the predicted phenomena, we introduce 


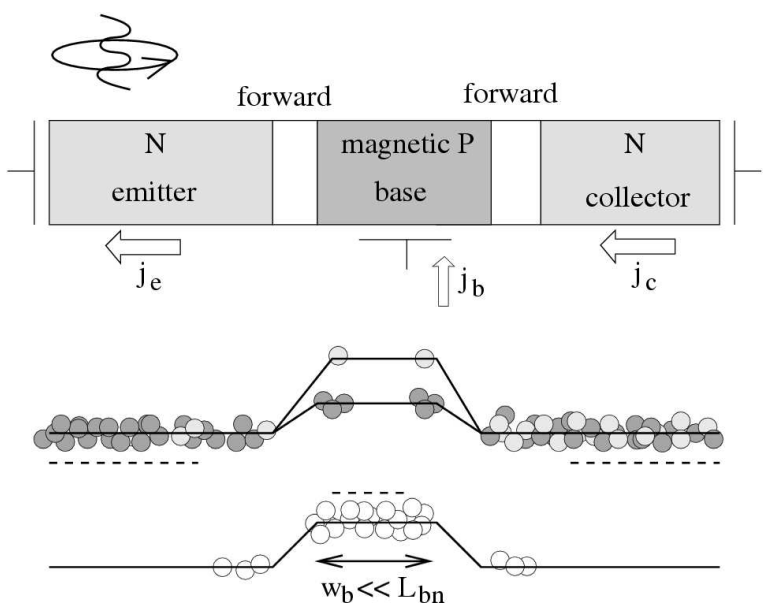

Fig. 2. Magnetic bipolar transistor. The upper figure shows the scheme used in the text. The emitter and collector are nonmagnetic $n$-doped regions, while the base is magnetic $p$-doped. The emitter can have a nonequilibrium spin injected optically or electrically. The currents flowing through different regions are identified. The lower figure shows the band diagram. The lower band is valence band, populated with holes (empty circles) which are assumed unpolarized. The upper band is the conductance band, with spin up (dark circles) and spin down (light circles) electrons. The conductance band is spin split in the base, indicating equilibrium spin polarization. In the saturation regime there is a forward bias (here taken equal) at both junctions. In the conventional case this means that the collector and emitter electron currents are the same, indicated by the equal drop of the chemical potential (dashed lines). In MBT's the chemical potential depends on spin, giving rise to spin-charge coupling.

below equations for the currents flowing through the different regions (see Fig. 2), and their relations to the excess (nonequilibrium) carrier densities. We then use simplifying assumptions to derive the currents and the current gain in the saturation regime. We will also present a numerical calculation based on the full analytical theory with no further simplifications.

The charge currents flowing in the emitter $\left(j_{\mathrm{e}}\right)$, collector $\left(j_{\mathrm{c}}\right)$, and base $\left(j_{\mathrm{b}}\right)$ can be written in the form usual for nonmagnetic bipolar transistors [14]:

$$
\begin{aligned}
& j_{\mathrm{e}}=j_{\mathrm{gb}}^{n}\left[\frac{\delta n_{\mathrm{be}}}{n_{0 b}}-\frac{1}{\cosh \left(w_{\mathrm{b}} / L_{n \mathrm{~b}}\right)} \frac{\delta n_{\mathrm{bc}}}{n_{0 \mathrm{~b}}}\right]+j_{\mathrm{ge}}^{p} \frac{\delta p_{\mathrm{eb}}}{p_{0 \mathrm{e}}}, \\
& j_{\mathrm{c}}=j_{\mathrm{gb}}^{n}\left[-\frac{\delta n_{\mathrm{bc}}}{n_{0 \mathrm{~b}}}+\frac{1}{\cosh \left(w_{\mathrm{b}} / L_{n \mathrm{~b}}\right)} \frac{\delta n_{\mathrm{be}}}{n_{0 \mathrm{~b}}}\right]-j_{\mathrm{gc}}^{p} \frac{\delta p_{\mathrm{cb}}}{p_{0 \mathrm{c}}} .
\end{aligned}
$$

The base current (for the signs of the currents see Fig. 2) is

$$
j_{\mathrm{b}}=j_{\mathrm{e}}-j_{\mathrm{c}} .
$$


The equilibrium electron density in the base is $n_{0 \mathrm{~b}}$, while the excess density in the base close to the junction with the emitter (collector) is $\delta n_{\mathrm{be}}\left(\delta n_{\mathrm{bc}}\right)$. Similar notation is used for the hole densities $p$. The electron diffusion length through the base of width $w_{\mathrm{b}}$ is $L_{n \mathrm{~b}}$. The electron contributions to the currents are proportional to the electron generation current in the base, which is

$$
j_{\mathrm{gb}}^{n}=\frac{q D_{n \mathrm{~b}}}{L_{n \mathrm{~b}}} n_{0 \mathrm{~b}} \operatorname{coth}\left(\frac{w_{\mathrm{b}}}{L_{n \mathrm{~b}}}\right) .
$$

Here $D_{n \mathrm{~b}}$ stands for the electron diffusion coefficient in the base. Similarly, the hole contribution depends on the respective hole generation currents in the emitter $\left(j_{\text {ge }}^{p}\right)$ and collector $\left(j_{\text {ge }}^{p}\right)$. These two generation currents have expressions analogous to Eq. (4).

The difference between the conventional and magnetic transistor appears in the expressions for the nonequilibrium carrier densities. The excess electron density in the base close to the emitter $\left(\delta n_{\mathrm{be}}\right)$ and collector $\left(\delta n_{\mathrm{bc}}\right)$ are respectively [14]:

$$
\begin{aligned}
& \delta n_{\mathrm{be}}=n_{0 \mathrm{~b}} \mathrm{e}^{q V_{\mathrm{be}} / k_{\mathrm{B}} T}\left(1+\alpha_{0 \mathrm{~b}} \delta \alpha_{\mathrm{e}}\right), \\
& \delta n_{\mathrm{bc}}=n_{0 \mathrm{~b}} \mathrm{e}^{q V_{\mathrm{bc}} / k_{\mathrm{B}} T}\left(1+\alpha_{0 \mathrm{~b}} \delta \alpha_{\mathrm{c}}\right),
\end{aligned}
$$

which follow from generalizing the implications of the spin-voltaic effect (a particular form of a spin-charge coupling), studied previously in magnetic diodes [5,6]. In conventional transistors the excess densities are determined solely by the Boltzmann factors containing the base-emitter and base-collector biases $V_{\mathrm{be}}$ and $V_{\mathrm{bc}}$, respectively. In MBT's the excess densities depend also on the spin-charge factors containing the equilibrium spin polarization in the base $\alpha_{0 \mathrm{~b}}$ and nonequilibrium spin polarization in the emitter and collector, $\delta \alpha_{\mathrm{e}}$ and $\delta \alpha_{\mathrm{c}}$. The temperature is $T$. For no spin-charge coupling the above equations reduce to the well known formulas for electron injection through a depletion layer [15]. Since holes are unpolarized, their excess densities in the emitter and collector are

$$
\begin{aligned}
& \delta p_{\mathrm{e}}=p_{0 \mathrm{e}} \mathrm{e}^{q V_{\mathrm{be}} / k_{\mathrm{B}} T}, \\
& \delta p_{\mathrm{c}}=p_{0 \mathrm{c}} \mathrm{e}^{q V_{\mathrm{bc}} / k_{\mathrm{B}} T} .
\end{aligned}
$$

In what follows we apply Eqs. (1) and (2) to a particular case of MBT in the saturation regime.

\section{Magnetic bipolar transistor in the saturation regime}

In the saturation regime both the emitter-base and base-collector junctions are forward biased. We consider first a special case of equal forward biases, $V \equiv$ $V_{\mathrm{be}}=V_{\mathrm{bc}} \gg k_{\mathrm{B}} T$. We also assume that the emitter and collector doping is larger than the base one, so that $p_{0 \mathrm{e}}, p_{0 \mathrm{c}} \ll n_{0 \mathrm{~b}}$ (the equilibrium minority carrier density is inversely proportional to the doping density) and we will be able to 
neglect the hole contributions to the currents. Such doping is unusual, since, due to technological reasons, in conventional bipolar transistors the collector doping is small. We note that the normal (active forward) regime of transistor operation is a forward emitter-base bias $\left(V_{\mathrm{be}}>0\right)$ and a reverse base-collector bias $\left(V_{\mathrm{bc}}<0\right)$, to ensure amplification.

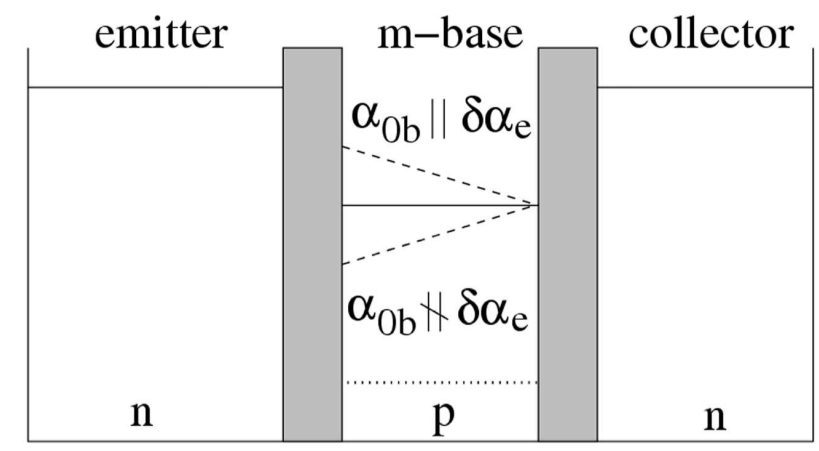

Fig. 3. Spatial profile of the electron density in an MBT in the saturation regime. In the $n$-doped emitter and collector the density is almost constant, at the donor doping level to ensure neutrality. In the magnetic base (m-base), the equilibrium density (dotted line) is very small, given by $n_{0 \mathrm{~b}}=n_{\mathrm{i}}^{2} / N_{\mathrm{ab}} \cosh \left(q \zeta_{\mathrm{b}} / k_{\mathrm{B}} T\right)$, where $n_{\mathrm{i}}$ is the intrinsic carrier density at a given temperature and $N_{\mathrm{ab}}$ is the acceptor doping in the base; $2 q \zeta_{\mathrm{b}}$ is the conduction band splitting in the base. If forward bias $V$ is applied, the electron density is raised to $n_{0 \mathrm{~b}} \exp \left(q V / k_{\mathrm{B}} T\right)$. If the same forward bias is applied to the emitter-base and collector-base junction, the electron density in the base will be almost uniform, giving rise to only small diffusion currents in the emitter and collector. If, however, the emitter spin is out of equilibrium, the injected electron density in the base will be $\delta n_{\mathrm{be}}=n_{0 \mathrm{~b}} \exp \left(q V / k_{\mathrm{B}} T\right)\left(1+\alpha_{0 \mathrm{~b}} \delta \alpha_{\mathrm{e}}\right)$, allowing an increase or decrease in $n$ depending on the relative orientation of $\alpha_{0 \mathrm{~b}}$ and $\delta \alpha_{\mathrm{e}}$ (dashed lines for parallel and antiparallel orientations). Significant current can flow in the collector.

Figure 3 illustrates the spatial profile of the electron density in a MBT. The excess electron density in the base determines how much current flows in the emitter and collector. If no spin-charge coupling is present, the density is almost uniform, creating only small diffusive currents. However, by changing the density at one junction by turning on spin polarizations, the density gradient gets larger leading to large collector and emitter currents. This is how spin induces current in MBT's. On the other hand, since the contributions from the spin-charge couplings to $j_{\mathrm{e}}$ and $j_{\mathrm{c}}$ are similar, they cancel in the base current $j_{\mathrm{b}}=j_{\mathrm{e}}-j_{\mathrm{c}}$. This is how the base current remains small, leading to large spin-induced current gain for small signal amplifications.

Let us take the collector current first. Substituting for the nonequilibrium electron density in Eq. (2), the following expression is obtained in the narrow base 
approximation $\left(w_{\mathrm{b}} \ll L_{n \mathrm{~b}}\right)$ :

$$
j_{\mathrm{c}}=j_{\mathrm{gb}}^{n} \mathrm{e}^{q V / k_{\mathrm{B}} T}\left[\alpha_{0 \mathrm{~b}}\left(\delta \alpha_{\mathrm{e}}-\delta \alpha_{\mathrm{c}}\right)-\frac{w_{\mathrm{b}}^{2}}{2 L_{n \mathrm{~b}}^{2}}-\frac{j_{\mathrm{gc}}^{p}}{j_{\mathrm{gb}}^{n}}\right] .
$$

If either the equilibrium or the nonequilibrium polarization vanishes, the charge current flowing through the collector is of the order of $j_{\mathrm{g}} \exp \left(q V / k_{\mathrm{B}} T\right) \max \left(w^{2} / L^{2}, j_{\mathrm{gc}}^{p} / j_{\mathrm{gb}}^{n}\right)$. This current is small in the narrow-base limit and in the limit of large collector doping. If a significant spin polarization is present, the current is much greater, on the order of $j_{\mathrm{g}} \exp \left(q V / k_{\mathrm{B}} T\right)$. Similar considerations hold for the emitter current.

Assuming that $\alpha_{0 \mathrm{~b}} \delta \alpha_{\mathrm{e}(\mathrm{c})} \gg w^{2} / L^{2}, j_{\mathrm{gc}}^{p} / j_{\mathrm{gb}}^{n}$, the important qualitative effect of Eq. (9) is that

$$
j_{\mathrm{c}} \sim \alpha_{0 \mathrm{~b}}\left(\delta \alpha_{\mathrm{e}}-\delta \alpha_{\mathrm{c}}\right) .
$$

Let us suppose there is a nonequilibrium spin either in the emitter or in the collector (or in both). The sign of the collector current can be changed by switching the magnetic field (changing $\alpha_{0 \mathrm{~b}}$, or changing the sign of the nonequilibrium spin polarizations). Since $j_{\mathrm{gb}}^{n}$ is usually known, measurements of $j_{\mathrm{c}}$ can directly give the product $\alpha_{0} \delta \alpha_{\mathrm{e}(\mathrm{c})}$ and thus directly detect the presence of nonequilibrium spin. On the other hand, the transistor functions as Johnson's spin switch: by changing the relative orientation of the equilibrium and nonequilibrium spins, $j_{\mathrm{c}}$ changes sign.

Another marked difference from the conventional bipolar transistor in the saturation regime is the emergence of a current gain. Current gain $\beta$, defined as

$$
\beta=\frac{j_{\mathrm{c}}}{j_{\mathrm{b}}}
$$

measures the amplification efficiency of the transistor. In the active forward regime $\beta$ is typically 100 . In the saturation regime $\beta$ becomes small, on the order of one. In MBT's, however, $\beta$ is large even in the saturation regime. Indeed, using our assumptions of magnetic and narrow base, and large emitter and collector dopings, the current gain is

$$
\beta=\frac{j_{\mathrm{gb}}^{n}}{j_{\mathrm{ge}}^{p}+j_{\mathrm{gc}}^{p}}\left[\alpha_{0 \mathrm{~b}}\left(\delta \alpha_{\mathrm{e}}-\delta \alpha_{\mathrm{c}}\right)\right] .
$$

The current gain is large because the prefactor, the ratio of the electron and hole generation currents is large, $\sim n_{0 \mathrm{~b}} / p_{0 \mathrm{e}(\mathrm{c})}$. The gain becomes small (on the order of one) if either of the spin polarizations vanishes. The remarkable fact is that in the saturation regime the current gain can even be negative.

The above approximations should be a robust description of the actual behavior in MBT's. Using our analytic theory [14] for ideal MBT's, without making any further assumptions, we show $\beta=\beta\left(\alpha_{0 \mathrm{~b}}\right)$ in Fig. 4 for a realistic MBT with generic room temperature materials parameters, one set borrowed from $\mathrm{Si}$ (main 
graph), one from GaAs (inset). The geometry and the doping profiles are the same. The emitter and the base have nominal lengths of $2 \mu \mathrm{m}$, the base is $1 \mu \mathrm{m}$. The emitter and collector donor densities are $10^{17} / \mathrm{cm}^{3}$, the base acceptor density is $10^{16} / \mathrm{cm}^{3}$. The applied forward voltages are 0.5 volts. Other parameters, such as diffusion coefficients and spin relaxation times, are given in Ref. [14]. The narrow base approximation is well valid for the Si-like case, where $L_{n \mathrm{~b}} \approx 30 \mu \mathrm{m}$, while it is not generally valid for our GaAs-like case, in which $L_{n \mathrm{~b}} \approx 3 \mu \mathrm{m}$. The results for Si show efficient spin control of the current gain, with the magnitude of $\beta$ similar to the normal active regime. In GaAs the gain is small, with the magnitude as in conventional bipolar transistors, although the sign of $\beta$ is still changed upon changing $\alpha_{0 \mathrm{~b}}$. The results of the full analytic theory are consistent with the approximate formula Eq. (12).

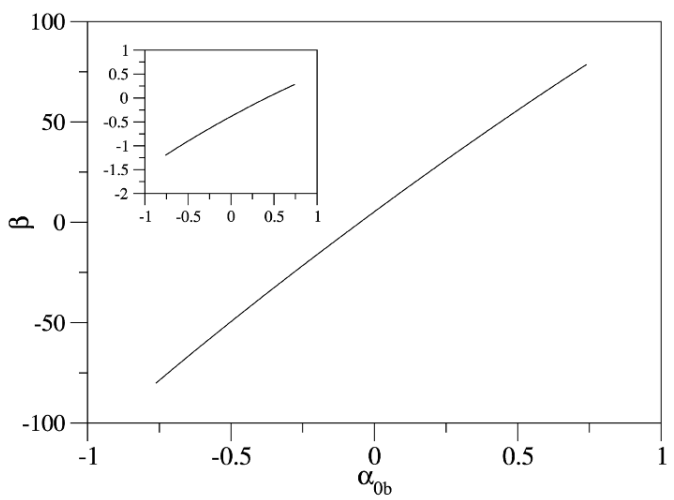

Fig. 4. Spin-induced current gain of MBT's as a function of the equilibrium spin polarization in the base $\alpha_{0 \mathrm{~b}}$ in the saturation regime. The nonequilibrium spin polarization is $\delta \alpha_{\mathrm{e}}=10 \%\left(\delta \alpha_{\mathrm{c}}=0\right)$. The main graph is for Si-like material parameters, the inset is for GaAs-like material parameters. The geometry is the same in both cases. Gain is less significant in the GaAs-like case, due to the breakdown of the narrow base assumption. The remedy would be to make the base narrower.

Finally, we ask what happens if the biases are unequal, $V_{\mathrm{be}} \neq V_{\mathrm{bc}}$. Let us suppose that $V_{\mathrm{bc}}=V_{\mathrm{be}}+\Delta V$. The above effects are valid for $\Delta V \ll k_{\mathrm{B}} T$ (perhaps if $\Delta V \leq 0.1 k_{\mathrm{B}} T$ ). However, one can tune $\Delta V$ in order to maximize the spin effects. It is straightforward to show, for example, that in the narrow-base approximation if

$$
\mathrm{e}^{-q \Delta V / k_{\mathrm{B}} T}=1+\frac{w_{\mathrm{b}}^{2}}{2 L_{n \mathrm{~b}}^{2}}+\frac{j_{\mathrm{gc}}^{p}}{j_{g \mathrm{~b}}^{n}}
$$

the collector current obeys Eq. (10). This relaxes the assumption of large collector doping and makes the predictions more robust. However, even if the spin control of $j_{\mathrm{c}}$ is not possible because the base is wide or the collector and acceptor doping are 
small, the spin effects (and thus spin injection and detection) can be observed in quantities such as $j_{\mathrm{c}}\left(\alpha_{0 \mathrm{~b}}\right)-j_{\mathrm{c}}\left(-\alpha_{0 \mathrm{~b}}\right)$, where the spin-independent parts cancel out.

\section{Conclusions}

We have described a novel phenomenon of spin-induced current gain in magnetic bipolar transistors in the saturation regime with similar emitter-base and base-collector forward bias voltages. Further crucial assumptions include narrow base, to make carrier diffusion through the base efficient, and small base acceptor doping, to neglect the hole currents. The transistor's collector and emitter currents can be switched by changing the relative orientation of the equilibrium spin polarization in the base and the nonequilibrium spin polarization in either the emitter or the collector. The gain also can change sign. In contrast to the conventional bipolar transistor, the current gain can be as large as in the active forward regime. These effects should be useful for sensing magnetic fields and for the spin control of current amplification. They also allow for measurements and detection of nonequilibrium spin densities in semiconductors, which should be useful especially in semiconductors in which optical spin detection is ineffective. There has been steady experimental progress in recent years towards bipolar (with respect to charge) spintronics [7, 18-21], which together with the exciting theoretical predictions warrants future investigations of the subject.

\section{Acknowledgments}

We are grateful to S. Das Sarma for useful discussions. I.Ž. acknowledges the National Research Council for financial support. This work was funded by DARPA, the NSF-ECS, and the US ONR.

\section{References}

[1] R.H. Silsbee, Bull. Magn. Reson. 2, 284 (1980).

[2] M. Johnson, R.H. Silsbee, Phys. Rev. Lett. 55, 1790 (1985).

[3] M. Johnson, J. Magn. Magn. Mater. 156, 321 (1996).

[4] M. Johnson, Science 260, 320 (1993).

[5] I. Žutić, J. Fabian, S. Das Sarma, Phys. Rev. Lett. 88, 066603 (2002).

[6] J. Fabian, I. Žutić, S. Das Sarma, Phys. Rev. B 66, 165301 (2002).

[7] I. Žutić, J. Fabian, S.D. Sarma, Rev. Mod. Phys. 76, 323 (2004).

[8] J. Fabian, I. Žutić, S. Das Sarma (2002), cond-mat/0211639.

[9] Optical Orientation, Eds. F. Meier, B.P. Zakharchenya, North-Holland, New York 1984.

[10] R. Fiederling, M. Kleim, G. Reuscher, W. Ossau, G. Schmidt, A. Waag, L.W. Molenkamp, Nature 402, 787 (1999). 
[11] B.T. Jonker, Y.D. Park, B.R. Bennett, H.D. Cheong, G. Kioseoglou, A. Petrou, Phys. Rev. B 62, 8180 (2000).

[12] D.K. Young, E. Johnston-Halperin, D.D. Awschalom, Y. Ohno, H. Ohno, Appl. Phys. Lett. 80, 1598 (2002).

[13] X. Jiang, R. Wang, S. van Dijken, R. Shelby, R. Macfarlane, G.S. Solomon, J. Harris, S.S.P. Parkin, Phys. Rev. Lett. 90, 256603 (2003).

[14] J. Fabian, I. Žutić, Phys. Rev. B 69, 115314 (2004).

[15] W. Shockley, Electrons and Holes in Semiconductors, D. Van Nostrand, Princeton 1950.

[16] J. Fabian, I. Žutić, S.D. Sarma, Appl. Phys. Lett. 84, 85 (2004).

[17] N. Lebedeva, P. Kuivalainen, J. Appl. Phys. 93, 9845 (2003).

[18] M. Kohda, Y. Ohno, K. Takamura, F. Matsukura, H. Ohno, Jpn. J. Appl. Phys. 40, L1274 (2001).

[19] E. Johnston-Halperin, D. Lofgreen, R.K. Kawakami, D.K. Young, L. Coldren, A.C. Gossard, D.D. Awschalom, Phys. Rev. B 65, 041306 (2002).

[20] F. Tsui, L. Ma, L. He, Appl. Phys. Lett. 83, 954 (2003).

[21] Y. Ohno, I. Arata, F. Matsukura, K. Ohtani, S. Wang, H. Ohno, Appl. Surf. Sci. 159-160, 308 (2000). 